\title{
Estructura agraria: lógicas disimiles en la concepción del territorio
}

\author{
Agrarian Structure: Different Fields of Logic on the Concept of \\ Territory
}

Wilson González Santos ${ }^{1}$

Para citar este artículo utilice el nombre completo así:

González, W. (2016). Estructura agraria: lógicas disimiles en la concepción del territorio. Perspectiva Geográfica, 21(1), 111-126.

\section{Resumen}

El desarrollo, como estrategia que mitiga el conflicto, es la apuesta dominante en los procesos de intervención en el espacio rural pero se ignora que la estructura agraria, como fenómeno que evidencia la cuestión agraria, se ha construido con el permanente pulso entre conflicto y desarrollo. El documento hace parte del referente conceptual para analizar las categorías conflicto y desarrollo en el territorio campesino. Se propone la trialéctica espacial como alternativa para comprender al campesinado y sus lógicas disímiles en sus procesos de intervención rural.

Palabras clave: campesino, conflicto, desarrollo, estructura agraria, rural.

1 Ingeniero Agrónomo, Magister en Desarrollo Rural. Filiación institucional: Docente Universidad Pedagógica y Tecnológica de Colombia (UPTC); Candidato a Doctor (UPTC-IGAC): Grupo de Investigación Competitividad y Desarrollo Local. País: Colombia. Correo electrónico: wilson.gonzalez@uptc.edu.co. 


\section{Abstract}

Development as a strategy to mitigate the conflict, is the dominant strategy for intervention processes in rural space. However, it ignores that the agrarian structure as a phenomenon which shows that the agrarian question, has been built with the constant interchange between conflict and development. This paper is part of the conceptual categories to analyze conflict and development in the rural territory. Spacial Trialectics are proposed as an alternative to understand the farmer and their different perspectives in rural intervention processes.

Keywords: farmer, conflict, development, agrarian infrastructure, rural. 


\section{Introducción}

De acuerdo con Teubal (2001), el afianzamiento de un sistema agroalimentario mundial orientado y dominado por grandes corporaciones transnacionales, además de las políticas de liberalización económica y de ajuste estructural aplicables al medio rural, son factores que han afectado sensiblemente la "nueva ruralidad" latinoamericana.

La política pública colombiana para el sector agrario no es ajena a este patrón, y promueve su inserción en el comercio internacional, con actividades agrícolas y pecuarias en función de ventajas comparativas y competitivas como, por ejemplo, el desarrollo de las cadenas agroexportadoras de cultivos permanentes. La hipótesis de dicha estrategia se basa en que el flujo y balance comercial es la bitácora para el crecimiento y desarrollo (Tobasura, 2009). En consecuencia, con la puesta en marcha de este modelo de desarrollo resulta una distribución bimodal en la estructura agraria (agroindustrial-campesina), bajo cuyo paradigma -el capitalismo agrario- el campesinado ${ }^{2}$ debe integrarse al mercado o desaparecer.

2 Partimos del concepto de campesino como una categoría social cuya unidad de producción es al
De acuerdo con Machado:

La dinámica del endeudamiento, bien conocida por los economistas, está conduciendo a acentuar aún más el modelo modernizante y a estrangular y a arrinconar más a la economía campesina. El Estado no parece tener otra alternativa a la de reforzar la agricultura de exportación, con los riesgos inflacionarios y los conflictos sociales que ello acarrea; y como no dispone de recursos fiscales para atender a la economía campesina, esta seguirá deteriorándose aún más (2003, p. 35).

No obstante, la lógica particular de la población campesina -actor representativo de la agricultura campesina- y su "vulnerabilidad", por el hecho de su aparente ineficiencia económica productiva, trasciende interpretaciones ligadas exclusivamente a la competitividad. En especial, para dicha población el espacio adquiere un significado es-

mismo tiempo unidad de consumo, con una activa participación del trabajo familiar y cuya finalidad principal es la reproducción familiar. No obstante, esta aseveración será solo una apuesta orientadora para nuestros propósitos. Con lo anterior y reconociendo la estrechez de la definición, los objetivos de la investigación obligan a un análisis mayor desde distintas perspectivas conceptuales y empíricas, el cual desarrolla posteriormente. 
pecial, que trasciende el mero concepto de contenedor físico, para convertirse en un objeto cargado de significados socio-culturales con un acento tanto objetivo como subjetivo, y al ser una construcción social, se encuentra sujeto a una dinámica en función de los procesos de articulación de quienes en él participan.

Las anteriores aseveraciones se traducen en una divergencia entre la bitácora inherente a las prácticas de poder para el sector rural y el quehacer de sus pobladores, en especial, de la comunidad campesina. En consecuencia, esta disímil comprensión del espacio rural produce un conflicto entre quienes orientan el desarrollo y quienes son afectados por este. De esta manera, el capital promueve no solo una particular territorialización, sino también recrea nuevas maneras de organización del espacio que, a su vez, resistidas por un grupo de actores, genera nuevos territorios.

A la luz de indicadores financieros, la aparente inviabilidad del sistema campesino, aunada a la composición de su estructura agraria, supondrían su desaparición del mismo ${ }^{3}$;

3 Un ejemplo de lo anterior constituye resultados de estudios realizados por González (2009a) (2009b) quien no obstante, la permanencia de sus actores ha obligado a poner en marcha estrategias de adaptabilidad, pero también de resistencia al modelo de desarrollo agrario propuesto. De esta manera, la estructura agraria resulta en una paradoja entre el modelo a imponer y la resistencia a su imposición.

El estudio forma parte de una síntesis de un texto reflexivo que sirvió de soporte teórico para la comprensión conceptual de los procesos de persistencia campesina en la investigación doctoral 'Dinámica campesina y estructura agraria'. Con una revisión de los estudios sobre la estructura agraria y su bimodalidad, se aborda la temática de territorio campesino como espacio de construcción social, con una lógica distinta a los procesos de intervención. El texto subraya la necesidad de un viraje en la aproximación a la comprensión del territorio campesino y propone la trialectica espacial como una alternativa pertinente.

advierte el comportamiento negativo de los indicadores económicos para la agricultura campesina en el sistema agroalimentario de la papa; este investigador evidencia esta cuestión al presentarse en el sistema ingresos netos negativos e índices estacionales por debajo de uno en la mayoría de los meses del año. 


\section{Desarrollo y discusión}

\subsection{La bimodalidad en la estruc- tura agraria}

El sector rural en Colombia, según Pérez (2001), experimenta su modernización a partir de la década de los años cincuenta, basado en una estructura bimodal soportada, de un lado, en un modelo empresarial altamente protegido, originado en el latifundio y especializado en la oferta de materias primas agroindustriales, y, por otro, en la presencia de un elevado número de unidades familiares campesinas, ubicadas en las zonas de ladera y dedicadas al suministro de alimentos a las poblaciones urbanas. Este tipo de modelo de desarrollo agropecuario, si bien se declara exitoso en lo económico, sustentado en el modelo proteccionista vigente hasta los años noventa, fue una de las principales causas de las asimetrías socioterritoriales generadas en el espacio rural colombiano.

Pérez (2001) considera que la política proteccionista desarrollada por el Estado dio origen a un modelo de desarrollo rural altamente inestable, inequitativo en la asignación de los recursos y poco competitivo en los mercados internacionales; destaca esta autora antes mencionada, que solo el café, las flores y el banano lograron sobrevivir a la apertura comercial de los noventa. Además, el modelo proteccionista no favoreció el desarrollo de la agricultura familiar campesina $y$ estimuló la ampliación de las fronteras agrícolas, lo que contribuiría a las migraciones del campo a la ciudad, las cuales se incrementan enormemente como consecuencia del conflicto armado y los desplazados que este genera.

La apertura económica y el modelo neoliberal, que se encuentra en total vigencia en el país, reporta fuertes impactos como resultado de la introducción de los ajustes estructurales de la fase neoliberal de la economía. De un lado, las corporaciones transnacionales y los capitalistas de la agricultura se benefician con la reducción del papel del Estado en el diseño de políticas públicas, así como de los incentivos a la agricultura a gran escala dirigida a la exportación. Por otro lado, se incrementa la lucha de los movimientos sociales del campo, que rechazan el modelo de desarrollo rural resultante de las políticas neoliberales, resistiendo de diferentes formas. 


\subsection{Antecedentes del concepto de estructura agraria}

La exploración de la producción académica sobre la estructura agraria y la dinámica campesina, en particular, en su fase hermenéutica, ha considerado la compilación de los trabajos en función de las distintas comprensiones de la conflictividad como "obstáculo" o coadyuvante en los procesos de intervención en el espacio rural ${ }^{4}$. Es importante precisar que esta postura corresponde con la perspectiva que se ha asumido como bitácora de la investigación: conflicto y desarrollo.

La reforma agraria - como manera de afectar la estructura agraria (Franco \& De los Ríos, 2011)- ha correspondido con la postura política que ha adoptado el estado para intervenir en el ámbito rural; en consecuencia, dicha postura se nutre de concepciones que soportan sus prácticas. Un aspecto interesante es la manera de abordar el conflicto en lo rural. Para el capitalismo agrario, desarrollo y conflicto son aspectos que deben tratarse de manera distinta: el desarrollo,

4 El aparte se ha construido en correspondencia con la fase heurística y hermenéutica de la investigación cuya bitácora son las categorías conceptuales conflicto y territorio. como mecanismo que permite solucionar el conflicto (Braun, Swaminathan, y Rosegrant, 2004). Lo anterior desconoce que el conflicto por la tierra ha estado íntimamente ligado al desarrollo rural; más aún, el conflicto, como resultante de procesos de adaptación o de resistencia al modelo de desarrollo agrario, explica la miscelánea de territorios que conviven en lo ru$\mathrm{ral}^{5}$. En este sentido, si se acepta la estructura agraria -como consecuencia de la intervención antrópica y de sus mecanismos de poder-, una revisión de la producción académica de esta temática, reproduce el acento político de quién o quiénes lo han propuesto:

No se puede desconocer que la objetividad y neutralidad de la ciencia es cuestionable si se tiene en cuenta que la separación del objeto de estudio omitiría que el investigador hace parte de su misma investigación, al ser parte activa como sujeto social y al fundamentarse en un lenguaje y un orden simbólico, contingente $y$, por ende, relativo (Fair, 2009, p. 62).

5 El autor prefiere usar el término rural, que "espacio rural". Considera que la promoción del desarrollo resultante de los conflictos entre los actores rurales territorializa y desterritorializa, con lo cual el espacio se carga permanentemente de significados, de territorios. 
Una revisión de los estudios sobre la estructura agraria indica el viraje en el núcleo central de su definición ${ }^{6}$. García (1982) señala que el núcleo de la estructura es la tierra, más específicamente, la propiedad y las relaciones que la afectan. Para este autor, la bimodalidad resulta cuando se forman dos aglomerados con tenencia de tierra disimiles y opuestas: propietarios con poca tierra, que conforman el minifundio, y otros con grandes extensiones que monopolizan gran parte del recurso. En este análisis, el conflicto es una implicación económica y una consecuencia de la estructura. En Colombia Franco y De los Ríos (2011) muestran que en el periodo en el cual predominó el concepto de García la reforma agraria se concentró en la tenencia de la tierra, como manera de afectar la estructura agraria. De acuerdo con lo anterior, en la década de los setenta, la tenencia de la tierra se convirtió en factor determinante para explicar la dinámica de la población rural latinoamericana (Pecht, 1976).

6 Si bien es cierto la estructura agraria es, per se, un constructo de naturaleza dinámica, asimilable al de sistema, y definida por las relaciones entre sus componentes, supone una relación primordial o "núcleo" que le da un carácter particular que la diferencia de otras estructuras (Machado, 2002).
Con la intensificación de las condiciones para el desarrollo del capitalismo, acrecentadas a fines del siglo XX, la concepción de la estructura agraria se modificó de manera sustancial. La nueva economía de mercado, la difusión tecnológica y la "competitividad" se convirtieron en un estribo sobre el cual se apoyaban las políticas agrarias. En consecuencia, según Machado (2002), la dicotomía latifundio-minifundio, dio paso a la economía empresarial-economía campesina $\mathrm{y}$, consecuentemente, la estructura agraria privilegió el mercado. Siguiendo a este último autor: "en el proceso de desarrollo y crecimiento de los niveles de ingreso per cápita, la estructura agraria evoluciona hacia una estructura agroindustrial o sistema agroindustrial (SAI)"’7.

No obstante, dicha bimodalidad no correspondería con la estructura agraria, al menos, si en su análisis se omite la dinámica inherente a su concepción. Bendini y Steimbreger (2010) introducen el concepto de territorialidad campesina para comprender "el desarrollo de estrategias que le permitan a un co-

$7 \quad$ Un ejemplo desde esta perspectiva se encuentra en el trabajo realizado en Chile por Andrade, Ríos y Torres (2012). 
lectivo resistir como campesinos $y$ permanecer en el territorio en un escenario de expansión concentrada del capital" (Andrade, Rios, y Torres, 2012, p. 62). Como se puede inferir, la territorialidad se da en un contexto $y$, se construye por actores y sus prácticas, pero es una categoría dinámica. Con lo anterior, entender la estructura agraria es comprender los procesos que la originaron, sus fuerzas, sus presiones, sus actores y su dialéctica de cambio en el tiempo.

Rosati (2011) trabaja el concepto de estructura social agraria en la provincia del Chaco (Argentina), a partir de la diferenciación social, explicada, en parte, por procesos de índole tecnológico y productivo, pero esencialmente por el impacto de la sojanización y la crisis algodonera. Este autor muestra la expresión territorial de estos procesos, por medio del comportamiento de indicadores, a partir de datos transversales censales. García (2012) indica que para el análisis de la estructura agraria es vital comprender los eventos ocurridos que afectaron la construcción del territorio. Jong (2008), desde un análisis regional cuestiona el principio de regulación de mercado (soporte del capitalismo agra- rio $)^{8}$, que, según el autor, subyace a la posición -de poder- que cada agente del proceso productivo ejerce en el momento.

Con lo anterior, se puede inferir que la estructura agraria es producto del modelo de desarrollo imperante, en este sentido, trascienden las concepciones que, si bien es cierto entienden sus relaciones, no la apalancan a las estrategias de intervención (García, 2012). Tampoco se inscribe, de manera estricta, a los enfoques que pretenden - definirla en función de los componentes propios del mercado (Machado, 2002) El trabajo apunta a definirla en un escenario donde el capitalismo agrario y la cuestión agraria son los actores principales de la conflictividad (Fernandes, 2004).

\section{Cuestión agraria y territorio campesino}

Según Valdés, la cuestión agraria:

Históricamente se ha definido como el complejo de causas y condiciones que caracterizan la situación de la sociedad rural y su lugar en la estructura y procesos de la sociedad global. En las

8 El paréntesis es del autor. 
sociedades subdesarrolladas, el problema agrario es un componente esencial de la formación, dependencia y contradicciones, de manera que su posible solución se vincula inevitablemente al proceso general de cambios políticos, económicos y sociales (1997, p. 3).

Como mecanismo necesario para trabajar en la búsqueda de solución a la compleja y crítica situación que presenta el espacio rural en el mundo subdesarrollado y por esta vía transformar la esencia de la cuestión agraria en estos países, se han aplicado políticas de Reforma Agraria como parte de las transformaciones políticas, económicas y sociales, con objetivos y alcances que se han diferenciado por países, y con resultados en muchos casos contradictorios. Por otra parte, la cuestión agraria en Colombia, o en cualquier región del planeta, no debe ser vista ni analizada aislada del contexto económico global.

La cuestión agraria, como un problema estructural engendrado por el avance del capitalismo en la agricultura, tiene en su centro de desarrollo las contradicciones que emergen en las relaciones capital/ campesinado; cuando se analizan estas relaciones en el seno de la expansión del capitalismo como formación socioeconómica, se produce lo que Harvey (2004) define como acumulación por despojo, caracterizada por la desterritorialización de las relaciones sociales, fenómeno que lleva implícito la destrucción de puestos de trabajo, la precariedad de las relaciones laborales, la destrucción de los territorios campesinos e indígenas y la segregación y marginalidad urbana. La acumulación por despojo significa un estado avanzado de las relaciones capitalistas, que necesitan cada vez menos reproducir y mantener las relaciones no capitalistas en los territorios subordinados.

Por tanto, la cuestión agraria es, además de la expresión del conflicto presente entre el desarrollo capitalista y la sociedad que demanda sus derechos y que se expresa en sus diversas dimensiones (política, económica, social y ambiental), un problema territorial. En el siguiente aparte se revisan las aproximaciones conceptuales para comprender la persistencia campesina.

\subsection{Territorio y persistencia cam- pesina}

Según González (2015) los discursos anticampesinistas que divulga- 
ban la desaparición del campesinado, por su aparente ineficiencia económica, resultan inadecuados, si se reconoce que la población rural latinoamericana muestra una inusitada permanencia.

La persistencia campesina ha sido explicada desde distintas escuelas de pensamiento. La línea chayanoviana propone que la lógica interna de este grupo humano le permite no solo resistir, sino reproducirse ante los embates del capitalismo. En especial, la producción agrícola familiar, de la cual el campesino es su principal representante, es simultáneamente una unidad de producción y de consumo. Ellis (citado por Boltvinik, 2012) indica que, para esta línea de pensamiento, los campesinos tienen algunas características que soportan su persistencia y reproducción, entre las cuales se destacan:

- el control sobre los medios de producción

- la especial racionalidad en la cual la reciprocidad y la economía moral soportan sus decisiones

- factores demográficos en contravía a la concentración de la tierra, dada la subdivisión en las herencias
- la flexibilidad en la cantidad de trabajo invertido en concordancia con el mayor o menor uso de mano de obra familiar

Con lo anterior, la lógica campesina difiere de la del sector agroindustrial en función de su racionalidad: el campesino para su supervivencia maximiza fundamentalmente su renta agrícola familiar y no su ingreso neto (Nuñez \& Díaz, 2006; González, 2015).

Por otra parte, la línea marxista sustenta que los procesos de diferenciación social resultarán en la desintegración de los campesinos, bien sea en agricultores capitalistas o trabajadores asalariados rurales (Boltvinik, 2012; Mora, 2008); sin embargo, desde esta escuela es posible explicar también la persistencia campesina. Según Ellis (1996), el capital es un motor que empuja a los campesinos a su reproducción simple, por la captura del excedente generado y la disminución del valor de su trabajo, como consecuencia de las innovaciones que disminuyen el precio de sus bienes. Como sustenta Boltvinik (2012), el patrón estacional de trabajo, propio de las actividades agropecuarias desarrolladas por los campesinos, es la bitácora que sostiene su te- 
sis, que el mismo autor sintetiza de la siguiente manera: "el capitalismo agrícola sólo puede existir en simbiosis con campesinos pobres, dispuestos a (y urgidos de) vender su fuerza de trabajo algunos días al año" (p. 24).

Un análisis de los enfoques propuestos permite comprender que la comprensión de la persistencia del campesino se sustrae a la aproximación conceptual con la cual se aborde; esta situación genera, necesariamente, limitaciones en función de abstracciones de la realidad propias de un marco conceptual determinado. Reconociendo lo anterior, y en consonancia tanto con el aparataje metodológico (análisis dialéctico actores sistema) como con la concepción del investigador respecto al cambio paradigmático, el estudio propone abordar al campesinado como un colectivo que, si bien, como propone Chayanov, tiene una dinámica que le ha permitido perpetuar su supervivencia, ha sido afectado sensiblemente por los embates y contradicciones del capital, que ha amenazado su espacio desterritorializando y recreando nuevos territorios en función de su lógica. Con lo anterior, y reconociendo que la tierra para el campesino la tierra es un espacio que trasciende su caracterización física y objetiva, la embestida del capitalismo agrario ha propiciado que lógicas disímiles al capital, como las propias del colectivo campesino, propicien mecanismos para resistir -consciente e inconscientementeen aras de su persistencia ${ }^{9}$.

\subsection{Una comprensión del territo- rio desde la trialéctica espacial}

El territorio además de ser un espacio que ha sido valorizado instrumentalmente (bajo el aspecto ecológico, económico o geopolítico), también lo es culturalmente. En el proceso de valorización, la interacción social diaria entre los habitantes permite que el territorio sea, significado, transformado y vivido. En el territorio se inscriben las características de quienes lo habitan y por lo tanto también es

9 La economía moral de los campesinos ofrece elementos para explicar su persistencia. Si bien es cierto, características propias de su dinámica -bien expresadas por Chayanov- obedecen a racionalidades heterodoxas para la economía convencional y sustentan estrategias de supervivencia, también lo es que su precaria posición frente a las élites lo hacían no solo trasgredir lo moral, o mejor, violar lo "normativamente" aceptado, sino posibilitar el germen de la reacción ante el estamento dominante. Como señaló Larson (1992) en su crítica a la economía moral "En fin, en la historia como en la vida real la economía moral nunca fue rígida o muy estática" (p. 97). 
fuente de los recuerdos (Hernández, 2011, p. 3).

Para la comprensión del territorio rural es esencial trascender las interpretaciones cartesianas del espacio - propias de la geografía teorética cuantitativa ${ }^{10}$, , con aproximaciones que admitan no solo el estudio de eventos complejos, difícilmente reducibles por la metodología científica positivista, sino también propios de otra lógica (González, 2015) ${ }^{11}$.

Los estudios de geografía urbana desarrollados por Lefebvre (1974) son también una herramienta valiosa para comprender los procesos socio-territoriales existentes en el ámbito rural. Siguiendo Lefebvre, el espacio es un producto social; cada sociedad y, en consecuencia, cada modo de producción concibe su espacio particular. De acuerdo con Ramírez (2004), para Lefebvre existen dos espacios, el teórico y el práctico o empírico, es decir, el espacio como producto social incorpora, a su vez, las acciones

10 Influenciada por el positivismo, la geografía cuantitativa no solo se adscribe al modelo cientificista propio del positivismo, sino marca la entrada del espacio como objeto principal de estudio geográfico. (Delgado, 2003).

11 Los eventos en cuestión cargan un equipamiento de forma y contenido incapaz de ser estudiado por el enfoque científico convencional. sociales de individuos que actúan en él. Con esta premisa, Lefebvre propone que cada sociedad crea un espacio donde confluyen tres esferas en una trialéctica a menudo conflictiva pero indisociable.

Con lo anterior, las representaciones del espacio, o el espacio concebido por científicos y planificadores, en función de las relaciones de producción vigentes converge con el espacio de representación, o experimentado plenamente por los habitantes, y con las prácticas espaciales que integran el espacio percibido por las personas ${ }^{12}$. Particularmente, los espacios concebidos y vividos serían difícilmente comprendidos desde las herramientas cuantitativas geográficas, esto es, cartográficas ${ }^{13}$. Como se puede inferir, el territorio campesino, un espacio construido con elementos objetivos pero muy especialmente subjetivos que, a su vez, explican la singular racionalidad del grupo humano que lo conforma, puede comprenderse desde la óptica de la trialéctica espacial.

12 Una dialéctica socio-espacial es un enfoque para el análisis de las formaciones sociales capitalistas y de concertación social. En ningún momento, para transitar el espacio -por sí mismo -a un nivel de objeto científico ni de forma autónoma respecto al análisis de las formas de producción (Soja, 1980).

13 La cartografía utilizada predominantemente, desde la institucionalidad, y relacionada con el espacio cartesiano o limítrofe. 
La Tabla 1 muestra algunos de los eventos de la agricultura campesina entendidos en el ámbito de la triada de Lefebvre. Cabe destacar que esta aproximación aunada a la mirada puramente cientificista de las representaciones espaciales, toma en consideración prácticas subjetivas con alta significación, evidenciadas en los espacios vividos y percibidos.

Tabla 1. La trialéctica espacial y la agricultura campesina

\begin{tabular}{|l|l|}
\hline Espacio & Ejemplos de eventos \\
\hline $\begin{array}{l}\text { Práctica espacial } \\
\text { (Espacio percibido) }\end{array}$ & $\begin{array}{l}\text { Caminos reales que comunican a unidades } \\
\text { de minifundio }\end{array}$ \\
\hline $\begin{array}{l}\text { Representaciones del espacio (Espacio } \\
\text { concebido) }\end{array}$ & Cartografía temática de usos de suelo \\
\hline $\begin{array}{l}\text { Espacios de representación } \\
\text { (Espacio vivido) }\end{array}$ & $\begin{array}{l}\text { Casa comunal de la vereda: espacio de } \\
\text { reunión donde se desarrollan actividades } \\
\text { con una fuerte carga emotiva }\end{array}$ \\
\hline
\end{tabular}

Fuente: Elaboración propia con base en Urrejola (2005), Ramírez (2004) y Lefebvre (1974).

\section{Conclusiones}

Los modelos de desarrollo en el espacio rural no pueden ser ajenos a la complejidad de esta porción geográfica; en especial, actores como los campesinos, que históricamente han construido su territorio, tienen lógicas distantes a las propias de la política pública y a sus procesos de intervención en lo rural. Es necesario un marco conceptual que se aproxime a los imaginarios objetivos y subjetivos que soportan el territorio campesino. Reconocer que el espacio tiene un significado que trasciende su concepción cartesiana es una exigencia perentoria; de esta manera, es necesario proponer nuevas aproximaciones que se acerquen a las múltiples lógicas que lo han cimentado. La geografía, como ciencia comprometida con el estudio del espacio, aporta elementos valiosos para el desarrollo rural; en particular, la trialéctica de la espacialidad, al reconocer múltiples espacios, se acerca a la complejidad del mundo rural y permite un marco conceptual apropiado para la intervención en él. 


\section{Referencias}

Andrade, L., Rios, S., y Torres, G. (2012). Análisis prospectivo de la estructura agraria en Chile basado en un modelo de Dinámica de sistemas. X Congreso Latinoamericano de Dinámica de Sistemas, pp. 1-8. Buenos Aires.

Bendini, I., y Steimbreger, N. (2010). Dinámicas territoriales y persistencia campesina. Revista Transporte y Territorio, 3, 59-78.

Boltvinik, J. (2012). Pobreza y persistencia del campesinado. Mundo siglo XXI, 8(28), 19-39.

Braun, J., Swaminathan, M. S., \& Rosegrant, M. (2004). Agricultura, seguridad alimentaria, nutrición y los objetivos de desarrollo del milenio. Washington: IFPRI.

Delgado, O. (2003). Debates sobre el espacio en la geografía contemporánea. Bogotá: Universidad Nacional de Colombia: Unibiblos.

Ellis, F. (1996). Peasant Economics. Farm Households and Agrarian Development. Cambridge University Press.

Fair, H. (2009). Contribuciones desde el post-estructuralismo lacaniano al debate epistemológico sobre la objetividad y la neutralidad valorativa. Revista de filosofia, 27(63), 35-63.

Fernandes, B. (2004). Cuestión agraria: conflictualidad y desarrollo territorial. Seminario Lincoln Center Institute. Harvard University.

Franco, A., y De los Ríos, I. (2011). Reforma Agraria en Colombia: evolución histórica del concepto. Hacia un enfoque integral actual. Cuadernos de Desarrollo Rural, 8(67), 93-119.

García, A. (1982). Modelos operacionales de reforma agraria y desarrollo rural en América Latina. San José de Costa Rica: Fondo de Cultura Económica.

García, M. (2012). Análisis de las transformaciones de la estructura agraria hortícola platense en los últimos 20 años. La Plata, Buenos Aires: Universidad Nacional de la Plata.

González, W. (2015). Economía campesina y territorio en las políticas de desarrollo rural. Revista Guillermo de Ockham 13 (2), Artículo IN PRESS.

González, W. (2009a). Educación y entorno en la ingenieria agronómica. Tunja: Universidad Pedagógica y Tecnoloógica de Colombia.

González, W. (2009b). La permanencia de la cadena agroalimentaria de la papa en Colombia: una interpretación desde la economia campesina. En Tercer congreso colombiano de horticultura, p. 112. Bogotá: Sociedad Colombiana de Ciencias horticolas.

Harvey, D. (2004). El nuevo imperialismo: acumulación por desposesión. Buenos Aires: CLACSO. 
Hernández, L. (2011). Geografía de los territorios rurales. Revista catalana de geografia, 16(42), $1-7$.

Jong, G. (2008). Análisis regional, estructuras agrarias y estrategias de desarrollo regional en la fruticultura del Alto Valle de la cuenca del río Negro. Tesis doctoral en Geografía. Universidad Nacional de La Plata.

Larson, B. (1992). Explotación y economia moral en los andes del sur. Historia critica, 6, 75-97.

Lefebvre, H. (1974). The Production of Space. Londres: Blackwell.

Machado, A. (2002). De la estructura agraria al sistema agroindustrial. Bogotá: Universidad Nacional de Colombia, Unibiblos.

Machado, A. (2003). Ensayos sobre seguridad alimentaria. Bogotá: Universidad Nacional de Colombia.

Mora, J. (2008). Persistencia, conocimiento local y estrategias de vida en sociedades campesinas. Revista de Estudios Sociales, 29, 122-133.

Nuñez, I., y Díaz, S. (2006). Innovación en la comunidad y economía campesina. México: Congreso Iberoamericano de Ciencia, Tecnología, Sociedad e Innovación.

Pecht, W. (1976). Estructura agraria y dinámica de la población en Brasil y México. Demografía y economía, 10(2), 254-283.

Pérez, E. (2001). Hacia uma nueva visión de lo rural. En G. Giarraca (Comp.), ¿Uma nueva ruralidad em América Latina? pp. 17-29. Buenos Aires: Clacso

Ramirez, B. (2004). Lefebvre y la producción del espacio. Sus aportaciones a los debates contemporáneos. Revista Veredas 5 (8), 61-73.

Rosati, G. (2011). Desigualdades y estructura social: producción, reproducción y cambio. Buenos Aires: IV Jornada de jóvenes investigadores Gino Germani.

Teubal, M. (2001). Globalización y nueva ruralidad en América Latina. En Giarraca, N. (comp.): ¿Uma nueva ruralidad em América Latina?, pp. 45-64. Buenos Aires: Clacso.

Tobasura, I. (2009). De campesinos a empresarios: la retórica neoliberal de la política agraria colombiana. Agronomía, 17(1), 21-34.

Urrejola, L. (2005). Hacia un concepto de espacio en antropología. Santiago de Chile: Universidad de Chile.

Valdez, J. (1997). Procesos agrarios en Cuba. La Habana: Felix Varela. 
Recepción: 31 de agosto de 2015

Evaluación: 31 de marzo de 2016

Aprobación: 4 de abril de 2016 